those with and without Sentinel Node Biopsy (SNB) in the first procedure.

Methodology Retrospective cohort study involving 27 patients diagnosed with low-risk EC (ESMO-ESGO-ESTRO criteria) with surgical restaging due to upstage in the final histological result at the Hospital Universitario Donostia from April 2013 to September 2018. Surgical and oncological results were compared between patients who underwent hysterectomy and double adnexectomy without any additional procedure (SNB-) $\mathrm{n}=17$ and those who also had a pelvic and aortic SNB (SNB +) $n=10$. The main outcome evaluated in the study was intraoperative complications. Secondary outcomes were mean operative time, length of hospital stay, number of nodes obtained, Progression-Free Survival (PFS) and Overall Survival (OS).

Results The median duration of restaging surgery was 240 minutes (Q25 - Q75: 180 - 300) in the SNB(-) group, and 300 (Q25 - Q75: 247.5 - 330) minutes in the $\mathrm{SNB}(+)$ group, this difference being statistically significant (one-side $\mathrm{t}$-student test, $\mathrm{p}=0.0295$ ). With regard to intraoperative complications, there were $17.65 \%$ vs $40 \%$ respectively, all of them vascular, this difference being not significant. There were no statistical differences in length of hospital stay and number of pelvic nodes obtained. PFS and OS in both groups were the same.

Conclusion Women with EC who require lymph node restaging due to upstage and have previously undergone sentinel lymph node biopsy have greater surgical difficulty with longer duration of the procedure. The risk of complications is increased.We advise against performing a second re-staging surgery in patients sentinel node biopsy.

Disclosures No disclosures.

\section{HAVE WE IMPROVED UTERINE SEROUS CARCINOMAS MANAGEMENT THE LAST TWO DECADES? A SINGLE CENTER EXPERIENCE}

${ }^{1}$ Michail Liontos, ${ }^{2}$ Anna Svarna, ${ }^{2}$ Roubini Zakopoulou, ${ }^{2}$ Flora Zagouri, ${ }^{3}$ Charalampos Theofanakis, ${ }^{3}$ Nikolaos Thomakos, ${ }^{2}$ Dimitrios Haidopoulos, ${ }^{4}$ Alexandros Rodolakis, ${ }^{5}$ Maria Sotiropoulou, ${ }^{6}$ Meletios-Athanasios Dimopoulos, ${ }^{6}$ Efi Skafida, ${ }^{6}$ Maria Kaparelou, ${ }^{6}$ Konstantinos Koutsoukos. 'General Hospital of Athens 'Alexandras'; ${ }^{2}$ National and Kapodistrian Unviersity of Athens; Department of Clinical Therapeutics; ${ }^{3}$ University of Athens; Alexandra Hospital; 1st Department of Obstetrics and Gynecology; ${ }^{4}$ National and Kapodistrian University of Athens; 1st Department of Obstetrics and Gynecology; ${ }^{5}$ Department of Pathology, Alexandra Hospital; ${ }^{6}$ Department of Clinical Therapeutics, National and Kapodistrian University of Athens, Alexandra Hospital

\subsection{6/ijgc-2020-ESG0.76}

Introduction/Background Uterine Serous Carcinomas (USC) account for ten percent of endometrial cancers. Management of the disease is challenging due to its aggressiveness. Despite numerous clinical trials and advances in the molecular pathology of the disease it remains the most lethal uterine carcinoma. We undertook this study to determine changes in the management of the disease and the survival of our patients the last two decades.

Methodology Medical records of women with USC treated at Alexandra Hospital from 2000 to 2018 were retrospectively identified. Clinicopathological data, treatment and survival data were analyzed. Kaplan-Meier Survival curves were generated using Strata SE 15.1; survival differences were estimated using the long-rank test.
Results 121 patients with USC and complete clinical data were identified. Median age was 66.9 years. Pure serous carcinomas were diagnosed in 66 patients, while the remaining had mixed histologies. At diagnosis, 33 patients $(28.0 \%)$ had stage Ia disease, but also 28 patients had stage IV disease (23.7\%). 115 patients were treated with surgery including lymphadenectomy in 63 cases $(55.8 \%)$ and omentectomy in 83 cases $(73.5 \%)$. 64 patients $(52.9 \%)$ were treated from 1999 to 2009. There was no statistical difference in the stage distribution, omission of lymphadenectomy or omentectomy and type of adjuvant treatment among patients treated prior or after 2010. Also, for patients with stage I-III disease, eligible for adjuvant treatment, both disease free survival and overall survival (OS) per stage did not differ significantly between the two decades. Finally, in patients with de novo stage IV disease there was no improvement in either first line Progression Free Survival or OS between the two decades (mPFS 1st vs 2nd decade: 8.6 vs 5.9 months 95\% CI [1.5-15.7].[3.4-8.5] and mOS 18.4 vs 11.6 months 95\% CI [1.7-34.9].[0.0-25.1].

Conclusion Despite recent clinical trials, surgical and medical management of USCs had not changed significantly the last two decades in everyday clinical practice. This is reflected in the survival of the patients.

Disclosures Authors have no conflict of interest.

\section{ENDOMETRIAL CANCER: THE ROLE OF PROGNOSTIC FACTORS AND THEIR IMPACT ON RECURRENCE PATTERN}

${ }^{1}$ Giulia Parpinel, ${ }^{2}$ Luca Fuso, ${ }^{1}$ Maria Elena Laudani, ${ }^{1}$ Annalisa Carapezzi, ${ }^{2}$ Enrico Badellino, ${ }^{2}$ Nicoletta Petronio, ${ }^{3}$ Marisa Ribotta, ${ }^{1}$ Martina Barboni, ${ }^{2}$ Annamaria Ferrero, ${ }^{1}$ Paolo Zola. ${ }^{1}$ Sant'anna Hospital; Department of Surgical Sciences; ${ }^{2}$ Mauriziano Hospital; Department of Surgical Sciences; ${ }^{3}$ Aou Città Della Salute e Della Scienza; Department of Pathological Anatomy

\subsection{6/ijgc-2020-ESG0.77}

Introduction/Background The rate of recurrence of endometrial cancer is $11-19 \%$. It is related to different prognostic factors which define specific risk classes in order to decide for an adjuvant treatment. The objective of this study is to evaluate how prognostic factors influence the probability and pattern of recurrence.

Methodology This multicentric observational retrospective study was conducted on 552 patients treated for endometrial cancer between February 2011-2019. The considered parameters were: age, BMI (Body Mass Index), surgery, stage, LVSI (Lymphovascular Space Involvement), myometrial infiltration, histological grade, lymph node involvement, adjuvant therapy, relapse. DFS (Disease Free Survival) and OS (Overall Survival) were stratified by the presence or absence of prognostic factors.

Results The rate of recurrence was $14,7 \%$. Median time to recurrence was 15 months and 5 -years OS was $84 \%$. We observed an increase from 6 to $30 \%$ in the distance metastasis rate associated with positive LVSI. Monovariate analysis showed a correlation between DFS and advanced stage (O.R. II 6.7; III-IV 9.2 $\mathrm{p}=0,0001$ ), positive lymph nodes (O.R. 3.7; $\mathrm{p}=0,04)$, myometrial infiltration (O.R. $3.9 \mathrm{p}=0,0001)$ and LVSI (O.R. $3.5 \mathrm{p}=0,005)$. Similar results were observed considering $O S$ and grading $(p=0,002)$. We conducted a Cox 
multivariate analysis on the ESGO/ESMO/ESTRO risk class classification and results were statistically significant for both DFS $(p=0,003)$ and OS $(p=0,0001)$.

Conclusion Almost all the considered prognostic factors influence the presence of recurrence, but the stage is the most important factor while LVSI correlates with distance metastasis. The definition of the risk factors must be considered to develop targeted therapeutic pathways.

Disclosures The authors declare that the research was conducted in the absence of any commercial or financial relationship that could be construed as a potential conflict of interest.

\section{METFORMIN AS A PREVENTIVE AND THERAPEUTIC MODALITY IN ENDOMETRIAL CANCER: A SYSTEMATIC REVIEW AND META-ANALYSIS OF RANDOMIZED CONTROL TRIALS}

Anastasia Prodromidou, Sofia Lekka, Alexandros Fotiou, Victoria Psomiadou, Dimitrios Giannoulopoulos, Christos R lavazzo. Metaxa Memorial Cancer Hospital; Metaxa Memorial Cancer Hospital of Piraeus; Gynecologic Oncolog

\subsection{6/ijgc-2020-ESG0.78}

Introduction/Background Endometrial cancer (EC) is the most commonly diagnosed gynecological malignancy in the developed countries. Obesity, diabetes mellitus and infertility are some of the contributory factors. Some patients with EC wish to preserve their fertility or others have several comorbidities that contraindicate surgery. These groups of patients could benefit from a conservative treatment strategy such as the use of metformin. This agent is an option in women with increased EC risk as well as in those with atypical endometrial hyperplasia.

Methodology We evaluated the protective effects of metformin in EC patients, its preventive role in breast cancer and obese patients and its effectiveness, safety and efficacy in addition to progesterone monotherapy in treatment of fertility sparing candidates. We reviewed the literature and then conducted a meta-analysis of the relevant parameters. A total of 6 studies was included in the meta-analysis.

Results Comparing the pre-surgical treatment with metformin versus placebo, meta-analysis of mean difference in Ki-67 after treatment among two groups, revealed no difference (MD 7.10, 95\% CI -23.31 to 9.11, p=0.39). Meta-analysis of fertility sparing EC management with a combination of megestrol acetate (MA) and metformin (500 $\mathrm{mg}$ three times a day) in comparison with monotherapy with $160 \mathrm{mg}$ daily MA revealed no difference in either complete response or partial response rates (166 patients OR 2.94, 95\% CI 0.85 to 10.15 , $\mathrm{p}=0.09$ and 166 patients OR $0.76,95 \%$ CI 0.34 to 1.66 , $\mathrm{p}=0.49$, respectively). Regarding breast cancer survivors under tamoxifen, metformin was related with significantly reduced median endometrial thickness after 52 weeks of evaluation when compared to women in placebo group (2.3 mm vs 3.0 $\mathrm{mm}, \mathrm{p}=0.05)$.

Conclusion Metformin neither was found to have a preventative role against the development of endometrial cancer nor a beneficial one in addition to the progesterone monotherapy for EC fertility sparing candidates. However, metformin was found to be protective in breast cancer survivors under tamoxifen.

Disclosures Nothing to disclose.

\section{9} COMBITEC: MULTICENTRIC RETROSPECTIVE STUDY ON SENTINEL LYMPH NODE DETECTION BY COMBINED ICG + 99MTC VERSUS EXCLUSIVE ICG IN ENDOMETRIAL CANCER

${ }^{1}$ Vicente Bebia Conesa, ${ }^{2}$ Marc Barahona, ${ }^{3}$ Cristina Almansa, ${ }^{4}$ Pablo Padilla Iserte, ${ }^{2}$ Lola Marti, ${ }^{2}$ Ponce Sebastiá Ponce, ${ }^{3}$ Alvaro Tejerizo, ${ }^{4}$ Santiago Domingo, ${ }^{5}$ Antonio GilMoreno, ${ }^{6}$ Silvia Cabrera Diaz. ${ }^{1}$ Hospital Universitari Vall D'hebron; Gynecologic Oncology; ${ }^{2}$ Hospital Universitari de Bellvitge; Gynecologic Oncology; ${ }^{3}$ Hospital Universitario 12 de Octubre; Gynecology; ${ }^{4}$ Hospital Universitari La Fe; Gynecologic Oncology; ${ }^{5}$ Fundació Vall Hebron; Institut de Recerca; Gynecologic Oncology; ${ }^{6}$ Hospital Universitari Vall D'hebron; Gynecology; Gynecologic Oncology

\subsection{6/ijgc-2020-ESGO.79}

Introduction/Background Despite its extended use, there is scarce evidence about the combined use of $99 \mathrm{mTc}$-albumin nanocolloid $(99 \mathrm{mTc})$ and indocyanine green (ICG) for the detection of sentinel lymph node (SLN) in endometrial cancer, when compared to ICG alone. The aim of this study is to compare the detection parameters of both methods.

Methodology Multicentric retrospective study (November 2015-June 2020) including patients diagnosed with endometrial atypical hyperplasia or initial preoperative stage endometrial carcinoma (FIGO I-II) who underwent SLN biopsy by cervical injection of: a) ICG intraoperatively, or b) $99 \mathrm{mTc}$ preoperatively, and ICG intraoperatively (ICG $+99 \mathrm{mTc})$.

Results A total of 180 patients were included, 51\% ( $\mathrm{n}=92)$ in the ICG group and $49 \%(n=88)$ in the ICG $+99 m$ Tc group. $86.7 \%$ of the patients presented endometrioid histology, and $58.7 \%$ were preoperatively classified as low risk, according to the ESMO/ESGO/ESTRO criteria. The vast majority of the procedures (99.4\%) were performed by a minimally invasive approach. Both groups were comparable regarding their basal characteristics, except for a higher body mass index (27.6 vs. $30.3 \mathrm{~kg} / \mathrm{m} 2, \mathrm{p}=0.014)$ in the ICG $+99 \mathrm{mTc}$ group and a bigger proportion of robotic-assisted procedures $(54.4$ vs $29.6 \%$, $\mathrm{p}=0.001)$ in the ICG group.

Global detection rate was 92.8\% (IC 95\%: 88.0-95.7), without statistically significant differences among groups (ICG:94.6\% vs ICG+99mTc:90.9\%, p=0.344). No significant differences were observed in the pelvic bilateral mapping rate $(71.6 \%$, ICG: $70.7 \%$ vs ICG $+99 \mathrm{mTc}: 71.6 \%, \mathrm{p}=0.890)$ or the aortic mapping rate $(5.6 \%$, ICG: $8.7 \%$ vs ICG $+99 \mathrm{mTc}: 2.3 \%, \mathrm{p}=0.058)$.

When ICG $+99 \mathrm{mTc}$ was used, surgical procedures were 30 minutes longer when compared to ICG $(150$ vs $180 \mathrm{~min}$, $\mathrm{p}=0.003)$. In 12 patients $(6.7 \%)$ at least one positive SLN was found (ICG:9.8\% vs ICG+99mTc:3.4\%, p=0.164).

No significant differences were observed regarding the empty node packets rate or the number of SLNs retrieved per patient. There were no patients with a positive lymphadenectomy specimen and a negative SLN, thus sensitivity was $100 \%$.

Conclusion Combining preoperative $99 \mathrm{mTc}$ to intraoperative ICG did not improve SLN detection in endometrial cancer, but resulted in longer procedures.

Disclosures Nothing to disclose.

\section{PREDICTIVE ROLE OF RADIOMIC FOR POST-OPERATIVE COMPLICATIONS OF LYMPHADENECTOMY IN EC PATIENTS}

${ }^{1}$ Francesco Magni, ${ }^{2}$ Massimiliano Fambrini, ${ }^{2}$ rene Paternò, ${ }^{2}$ Virginia Taddei, ${ }^{2}$ Flavia Sorbi, ${ }^{2}$ Silvia Lucarini, ${ }^{2}$ Mazzoni Laura, ${ }^{2}$ Felice Petraglia. ${ }^{2}$ Careggi University Hospital; Gynecology and Obstetrics; ${ }^{2}$ Careggi University Hospital

10.1136/ijgc-2020-ESGO.80 